DOI: $10.4274 /$ jarem.galenos.2018.2019

J Acad Res Med 2020; 10(1): 97-9

\title{
Sitagliptin/Metformin Related Cutaneous Leukocytoclastic Vasculitis in a Patient with Type-2 Diabetes Mellitus
}

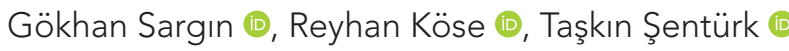 \\ Aydın Adnan Menderes University Faculty of Medicine, Department of Rheumatology, Aydın, Turkey
}

Cite this article as: Sargın G, Köse R, Şentürk T. Sitagliptin/Metformin Related Cutaneous Leukocytoclastic Vasculitis in a Patient with Type-2 Diabetes Mellitus. JAREM 2020;10(1): 97-9

\begin{abstract}
Small vessel vasculitis is characterized by the involvement of arterioles, capillaries, and venules. The most common cutaneous finding of small vessel vasculitis is palpable purpura, and urticaria, petechiae, erythema, vesicles, and pustules may also be seen. It may be related to malignancies, connective tissue diseases, infections, and drugs such as penicillin, sulphonamides, allopurinol, antithyroid drugs but it is often idiopathic. Drug-related vasculitis is seen in 7 to 21 days after the onset of the drug and systemic findings are usually absent. Both metformin and sitagliptin are frequently used in the treatment of diabetes. However, skin reactions to these drugs are rare. In this case report, our aim is to present a 70-year-old female patient with the diagnosis of type 2 diabetes mellitus, and metformin/sitagliptin-induced cutaneous leukocytoclastic vasculitis.
\end{abstract}

Keywords: Sitagliptin/metformin, cutaneous leukocytoclastic vasculitis, type 2 diabetes mellitus

\section{INTRODUCTION}

Leukocytoclastic vasculitis (LCV) is a small vessel vasculitis characterized by the involvement of arterioles, venules, capillaries with inflammation and necrosis. It may be related to malignancies $(<5 \%)$, connective tissue diseases $(15-20 \%)$, infections $(15-20 \%)$, and drugs such as penicillin, sulphonamides, allopurinol, and antithyroid drugs (10-15\%) but it is often idiopathic (50\%) (1). Also, tartrazine, insecticides, herbicide, diphenylhydantoin, acetylsalicylic acid, naproxen, and furosemide cause LCV (2). Skin biopsy is needed to diagnose LCV and to exclude mimicking causes, and drug-related LCV is an exclusion diagnosis. Dipeptidyl peptidase (DPP)-4 inhibitors are new generation oral anti-diabetic drugs developed for type 2 diabetes mellitus (DM) and the mechanism of action is to inhibit the degradation of incretins (3). Common side effects of DPP-4 inhibitors include nausea, abdominal pain, diarrhea, and rarely pancreatitis $(3,4)$. However, there are studies reporting that sitagliptin has no association with vomiting, nausea, and diarrhea (5).

Sitagliptin and its metabolites- related hypersensitivity reactions and generalized skin eruption have been reported $(3,4,6)$. However, LCV associated with sitagliptin is extremely rare (4). The most common side effects of metformin are dyspeptic symptoms such as flatulence, nausea, and vomiting but skin reactions are rare. Metformin-associated psoriasiform drug eruption and LCV have been reported in the literature $(6,7)$. Here, we aimed to present a female patient with metformin plus sitagliptin-induced cutaneous LCV.

\section{CASE PRESENTATION}

A 70-year-old female patient was admitted to our clinic with a complaint of rashes on her legs. On physical examination, the patient was morbidly obese and there were symmetrical

ORCID IDs of the authors: G.S. 0000-0002-3778-8351; R.K. 0000-0001-9017-8483; T.Ş. 0000-0002-1560-6764. 
erythematous papules and palpable purpura on both lower extremities (Figure 1). There was no medical history of smoking or alcohol use. She had hypertension for 15 years, type 2 DM for 14 years, asthma for 20 years, and was taking insulin aspart (fast-acting insulin analog), insulin detemir (long-acting human insulin analog), metformin, inhale glycopyrronium bromide (longacting muscarinic antagonist as a bronchodilator $\beta 1$ receptor blocker) and nebivolol (nitric oxide-potentiating vasodilator). Metformin was withdrawn and therapy was continued with sitagliptin/metformin due to impaired blood glucose regulation 10 days before. The blood results were as following: hemoglobin: $14.3 \mathrm{~g} / \mathrm{dL}$, hematocrit: 44,6\%, mean corpuscular volume: $92 \mathrm{fL}$, leukocyte: $9.230 / \mathrm{mm}^{3}$, platelet: $249.000 / \mathrm{mm}^{3}$, C-reactive protein: $34.4 \mathrm{mg} / \mathrm{L}$, sedimentation: $44 \mathrm{~mm} / \mathrm{h}$, Urinalysis and stool analysis were normal. The anti-nuclear antibody and anti-neutrophilic cytoplasmic antibody were negative. And, rheumatoid factor, anticyclic citrullinated peptide, immunoglobulins, and complement levels were within normal ranges. Hepatomegaly/hepatosteatosis was detected by abdominal ultrasonography and chest X-ray was normal. Left ventricular diameter and motions were normal by echocardiography. Skin biopsy revealed LCV with neutrophilic inflammation, fragmented neutrophilic nuclei, and fibrinoid necrosis. It was considered to be associated with sitagliptin. The suspected drug was discontinued and $0.5-1 \mathrm{mg} / \mathrm{kg}$ prednisolone was started. In the follow-up, the rashes of the patient disappeared within one week. Written informed consent was obtained from the patient.

\section{DISCUSSION}

In the histopathological examination of cutaneous vasculitis, neutrophilic transmural inflammation and fibrinoid necrosis,

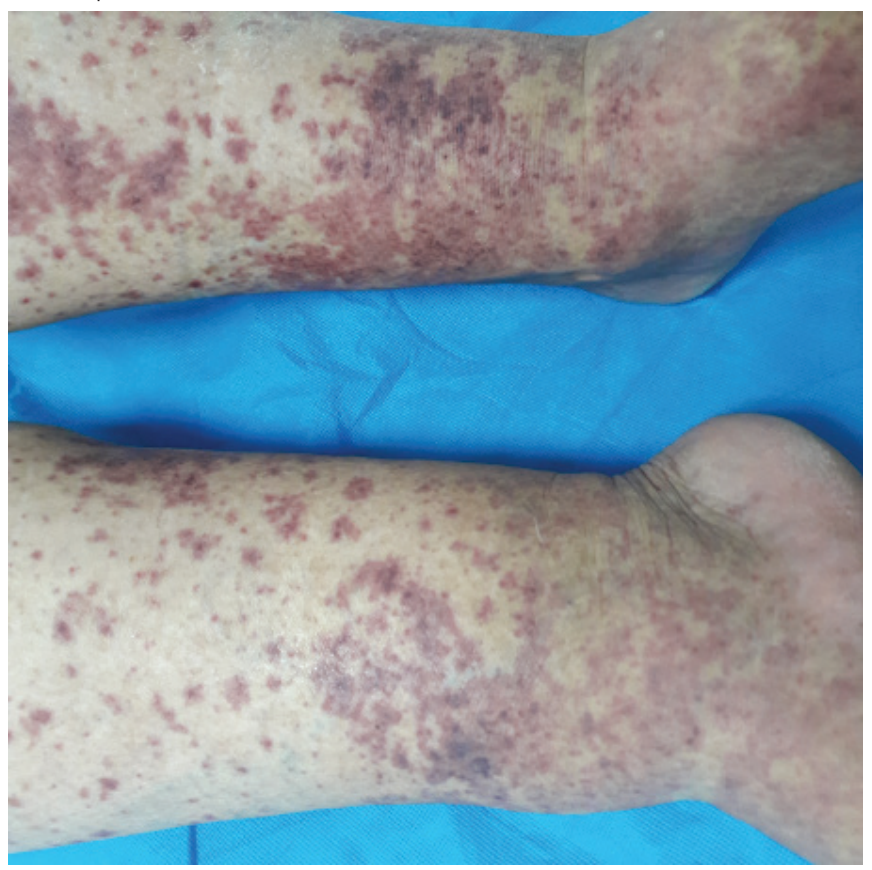

Figure 1. Symmetrical erythematous papules and palpable purpuras on both lower extremities extravasation of erythrocytes, granulocyte debris are seen on the vessel wall, and granulomatous or lymphocytic infiltration and immunoreactant deposition may also be seen according to etiology (1). Drug-related vasculitis is seen in 7 to 21 days after the onset of the drug and systemic findings are usually absent. Diagnosis is usually made by questioning time of exposure to the suspected drug and excluding other causes such as infections, connective tissue diseases and malignancies (1). We have not found infections, connective tissue diseases, and malignancies as underlying causes in our patient. Due to the recent addition of sitagliptin plus metformin to the treatment of the patient, it was thought to be drug-induced LCV.

Metformin is widely used in tip 2 DM. Rash, urticaria, bullous pemphigoid, psoriasiform drug eruption, LCV, lichen planus, and acute alopecia have been reported as metformin-induced skin eruptions $(7,8)$. In the literature, there are few cases on metformininduced LKV. Ben Salem et al. (8) described a case of LKV that developed after metformin in a 33-year-old female patient. She started self-treatment with metformin twice one month apart, but at both times, maculopapular rash and petechial lesions developed on her lower extremities after metformin $850 \mathrm{mg}$ treatment. Lesions resolved by discontinuation metformin and biopsy showed LCV.

Sitagliptin is a new generation oral antidiabetic drug used in the treatment of type $2 \mathrm{DM}$. Dyspeptic symptoms and hypersensitivity reactions may occur with sitagliptin $(3,4)$. Anaphylaxis and angioedema may occur due to the use of a DPP-4 inhibitor (4). Sitagliptin-associated skin and subcutaneous diseases were found to be 1.3 per 100 patient-years (3). It has been reported that gliptins may cause serious reactions such as toxic epidermal necrolysis and Steven Jonhson's syndrome (4).

There are also gliptin-related bullous pemphigoid case reports and case series (9). In a case report of Attaway et al. (9), 70 yearold male patients with type 2 DM developed bullous eruption and urticaria one year after the start of sitagliptin therapy. Skin biopsy revealed bullous pemphigoid, healed within two to three days with the discontinuation of sitagliptin and initiation of steroids. Authors did not find a strong association with bullous pemphigoid and other drugs of the patient (9). In the review of the case reports, $64 \%$ of the patients were using vildagliptin and $36 \%$ were using sitagliptin, the mean age was 72 years with male predominance and the mean interval between bullous pemphigoid and drug initiation was found to be 6 months (9).

Uçan et al. (10) reported sitagliptin-induced LCV in a 46-year-old male patient. He was followed up for 6 years with type $2 \mathrm{DM}$ and was using $2000 \mathrm{mg}$ of metformin and $100 \mathrm{mg}$ of sitagliptin was added to the treatment. Fifteen days after sitagliptin initiated, bilateral rashes appeared on his legs. There was no evidence of systemic involvement and skin biopsy revealed LCV. Within 10 days after the discontinuation of sitagliptin treatment, the patient's rashes disappeared. In another case, generalized skin eruption was reported in a patient using sitagliptin plus metformin (6). In a 66-year-old male with untreated type $2 \mathrm{DM}$, treatment was 


\section{REFERENCES}

started with sitagliptin $50 \mathrm{mg}+$ metformin $500 \mathrm{mg}$. Six months later a rash occurred on the upper limbs of the patient and then spread to the chest, back, abdomen and thigh. The skin lesions did not respond to steroid treatment but resolved immediately after sitagliptin discontinuation. The authors did not perform skin biopsy, and they considered sitagliptin-induced allergic reaction in their differential diagnosis. Other hypersensitivity reactions reported with sitagliptin are erythema, urticaria, and angioedema $(3,4)$.

\section{CONCLUSION}

Cutaneous vasculitis cases due to both metformin and sitagliptin are rare. The combination of sitagliptin/metformin is frequently used in the treatment of diabetes. It should be kept in mind that metformin plus sitagliptin may cause cutaneous LCV.

Informed Consent: Written informed consent was obtained from the patient.

Peer-review: Externally peer-reviewed.

Author Contributions: Concept - G.S., R.K., T.Ş.; Design - G.S., R.K., T.Ş.; Supervision - G.S., R.K., T.Ş.; Resources - G.S., R.K., T.Ş.; Data Collection and/or Processing - G.S., R.K., T.Ş.; Analysis and/or Interpretation - E.K.; Literature Search - G.S., R.K.; Writing Manuscript - G.S., R.K.; Critical Review - G.S., R.K., T.Ş.

Conflict of Interest: The authors have no conflict of interest to declare.

Financial Disclosure: The authors declared that this study has received no financial support.
1. Chung L, Fiorentino D. Cutaneous vasculitis. Orphanet encyclopedia 2005; 1-18

2. Ünübol M, Ceyhan C, Şentürk T, Tataroğlu C. Furosemide-induced cutaneous leucocytoclastic vasculitis: Case report. Turkiye Klinikleri 2011; 23: 79-82.

3. Raz I, Hanefeld M, Xu L, Caria C, Williams-Herman D, Khatami H, et al. Efficacy and safety of the dipeptidyl peptidase-4 inhibitor sitagliptin as monotherapy in patients with type 2 diabetes mellitus. Diabetologia 2006; 49: 2564-71.

4. Williams-Herman D, Engel SS, Round E, Johnson J, Golm GT, Guo H, et al. Safety and tolerability of sitagliptin in clinical studies: a pooled analysis of data from 10,246 patients with type 2 diabetes. BMC Endocr Disord 2010; 10:7.

5. Karagiannis T, Boura P, Tsapas A. Safety of dipeptidyl peptidase 4 inhibitors: a perspective review. Ther Adv Drug Saf 2014; 5: 138-46.

6. Nakatani K, Kurose T, Hyo T, Watanabe K, Yabe D, Kawamoto T, et al. Drug-induced generalized skin eruption in a diabetes mellitus patient receiving a dipeptidyl peptidase-4 inhibitor plus metformin. Diabetes Ther 2012; $3: 14$

7. Badr D, Kurban M, Abbas O. Metformin in dermatology: an overview. J Eur Acad Dermatol Venereol 2013; 27: 1329-35.

8. Ben Salem C, Hmouda H, Slim R, Denguezli M, Belajouza C, Bouraoui K. Rare case of metformin-induced leukocytoclastic vasculitis. Ann Pharmacother 2006; 40: 1685-7.

9. Attaway A, Mersfelder TL, Vaishnav S, Baker JK. Bullous pemphigoid associated with dipeptidyl peptidase IV inhibitors. A case report and review of literature. J Dermatol Case Rep 2014; 8: 24-8.

10. Uçan B, Topaloğlu O, Önder E, Çakal E, Karbek E, Öztürk Ünsal B, et al. Sitagliptin Induced Cutaneous Leukocytoclastic Vasculitis: A Case Report. Ortadogu Medıcal Journal 2012; 4: 199-201. 